\title{
A Comparative Study of LFM Reverberation Suppression Schemes
}

\author{
Jami Venkata Suman ${ }^{1}$ and J. Beatrice Seventline ${ }^{2}$ \\ ${ }^{1}$ Assistant Professor, Department of ECE, GMR Institute of Technology, \\ Rajam, India \\ ${ }^{2}$ Professor, Department of ECE, GITAM Institute of Technology, \\ Visakhapatnam, India \\ 1jami.venkatasuman@gmail.com
}

\begin{abstract}
The research work in this paper is based on, improving the target detection performance under reverberation scenario which is the most important issue when active sonar's are used for target detection. Reverberation is echo due to scatterers and boundaries in the water when the transmitted signal off. Reverberation is severely colored, correlated strongly with the emitted signal and non stationary in time and frequency domains. It is also frequency dependent in nature and the pulse length is more because it is doubly spread. Due to these difficulties matched filtering is ineffective. The motivation behind this paper is to suppress LFM reverberation, which is present while transmitting a Linear Frequency Modulated (LFM) signal. In this paper, the performance analysis of dechirping transformation based and Fraction Fourier Transform (FrFT) based parametric power spectrum estimation methods such as Autoregressive (AR), Moving Average (MA) and Autoregressive Moving Average (ARMA) prewhitening models were studied with respect to LFM reverberation data and results are presented. The simulation results with LFM reverberation data verify that the proposed dechirping transformation based and FrFT based MA and ARMA prewhitening models improves the target detection performance of active sonar systems.
\end{abstract}

Keywords: LFM Signal, Reverberation, AR prewhitening, MA prewhitening, ARMA prewhitening, Dechirping Transformation, Fractional Fourier Transform

\section{Introduction}

Over the last few years there has been an excellent development of signal processing and analysis techniques in applications of sonar and radar which improves target detection performance and extract the characteristics of the signal, if the signals are statistically time varying or non-stationary in nature. Target detection particularly in underwater scenario is one of the main problems in active sonar as the transmitted signal undergoes reverberation. When a signal is transmitted it undergoes multiple reflections by the surface and bottom interfaces or obstacles. When the target is close to one interface, the target echo is invisible in the reverberation resulting in a low signal to reverberation ratio (SRR). Reverberation is colored, frequency dependent, strongly correlated with the emitted signal and non-stationary in nature. The conventional method for detecting the low speed moving targets such as matched filtering is inefficient as reverberation is strongly correlated with signal. Recently, many researchers focus on developing antireverberation methods. In this process linear chirp signal is widely used because it has a good focusing property. Improved active sonar detection using autoregressive prewhiteners was presented by Kay [7] in which the reverberation in the current block is whitened based on modeling the reverberation in the previous block. But it is difficult to whiten LFM reverberation efficiently. An autoregressive (AR) prewhitener for LFM 
reverberation was suggested by Camillet [6] in which the reverberation in the current block was whitened through modeling the reverberation in the previous block. However, the method is based on the assumption of local stationary, and, actually, the frequency variation of LFM signal undermines the whitening performance. For providing a stationary frequency property to the LFM reverberation, Choi [2] improved the above mentioned method using dechirping transformation but this method may weaken the target echoes with low Doppler shift. An anti reverberation method based on FrFT was given by Chen [8] which attempts to enhance the target signal via a narrow band filter in a fractional Fourier domain, but it needs to search for the peak of the target signal before filtering. To avoid the uncertainty of target searching, Ruhang Wang [1] presented improved space time prewhitener for LFM reverberation using FrFT in which FrFT based space time prewhitening for enhancing the target signal by combining the space time prewhitening with FrFT.

In this paper, proposed first method uses dechirping transformation to inversely compensate the frequency chirp rate of the LFM signal. This is done for attaining a stationary frequency property of LFM reverberation in each data block. The stationary reverberation is cut in to blocks using a sliding window and the frequency response of current data block is modeled using the coefficients of the adjacent block as reference. Finally, using these coefficients, implement the inverse filter and well prewhiten the LFM reverberation of the current beam using parametric power spectrum estimation AR, MA and ARMA methods. The proposed second method is using FrFT theory. In order to provide a stationary reverberation, a slow time varying envelop of reverberation is computed. The stationary reverberation is cut into blocks using a sliding window and each block is whitened using parametric power spectrum estimation AR, MA and ARMA methods in fractional domain (u domain).

\section{Prewhitening using Conventional Methods}

Spectrum estimation of reverberation plays a main role in prewhitening process. Reverberation is highly non-stationary it is very difficult for us to estimate the spectrum precisely. Consider an emitted signal is LFM and a uniform linear array with B sensors. The detection problem is given below

$$
\mathrm{x}(\mathrm{t})=\mathrm{x} 1(\mathrm{t})+\mathrm{x} 2(\mathrm{t})+\mathrm{x} 3(\mathrm{t})
$$

where $x(t)$ is the received LFM reverberation signal, $x 1(t)$ is the target echoes, $x 2(t)$ is the reverberation or correlated noise and $x 3(t)$ is the uncorrelated noise. The received signal $\mathrm{x}(\mathrm{t})$ is divided into blocks using sliding windows in time domain. If the LFM reverberation data to be whitened is positioned at the $\mathrm{m}^{\text {th }}$ time block of $\mathrm{n}^{\text {th }}$ beam, given by $\mathrm{x}_{(\mathrm{m}, \mathrm{n})}(\mathrm{t})$, the AR coefficients $\left\{1, \mathrm{a}_{1}, \ldots \ldots, \mathrm{a}_{\mathrm{p}}\right\}$, MA coefficients $\left\{1, \mathrm{~b}_{1}, \ldots \ldots, \mathrm{b}_{\mathrm{q}}\right\}$ are extracted from modeling the reverberation of the adjacent beam $x_{(m, n-1)}(t)$ or $x_{(m, n+1)}(t)$ using a $p^{\text {th }}$ order AR model, $\mathrm{q}^{\text {th }}$ order MA model, $\left(\mathrm{p}^{\text {th }}, \mathrm{q}^{\text {th }}\right)$ order ARMA model respectively [1]. The reverberation is whitened by parametric (model-based) AR, MA and ARMA models in which the current block is prewhitened by using the adjacent block as reference signal [9].

The linear filter $\mathrm{H}(\mathrm{z})$ for generating the random process $\{\mathrm{x}(\mathrm{n})\}$ from the white noise sequence $\{\mathrm{w}(\mathrm{n})\}$ is also rational and is expressed as [10]

$\mathrm{H}(\mathrm{z})=\frac{\mathrm{B}(\mathrm{z})}{\mathrm{A}(\mathrm{z})}=\frac{\sum_{\mathrm{k}=0}^{\mathrm{q}} \mathrm{b}_{\mathrm{k}} \mathrm{z}^{-\mathrm{k}}}{1+\sum_{\mathrm{k}=1}^{\mathrm{p}} \mathrm{a}_{\mathrm{k}} \mathrm{z}^{-\mathrm{k}}}$

where $\{a k\}$ and $\{b k\}$ are the filter coefficients that determine the location of the poles and zeros of $\mathrm{H}(\mathrm{z})$. Both $\mathrm{H}(\mathrm{z})$ and its reciprocal $\frac{1}{\mathrm{H}(\mathrm{z})}$ is casual, stable and minimum phase linear system. Therefore, the random process $\{\mathrm{x}(\mathrm{n})\}$ individually represents the statistical properties of the innovation process $\{\mathrm{w}(\mathrm{n})\}$, and vice versa. 


\section{A. AR Model}

In this model, $\mathrm{b}_{0}=1, \mathrm{~b}_{\mathrm{k}}=0, \mathrm{k}>0$, the linear filter $\mathrm{H}(\mathrm{z})=\frac{1}{\mathrm{~A}(\mathrm{z})}$ is an all-pole filter and the difference equation for the input-output relationship is

$\mathrm{x}(\mathrm{n})+\sum_{\mathrm{k}=1}^{\mathrm{p}} \mathrm{a}_{\mathrm{k}} \mathrm{x}(\mathrm{n}-\mathrm{k})=\mathrm{w}(\mathrm{n})$

\section{B. MA Model}

In this model, $a_{k}=0, k \geq 1$, the linear filter $H(z)=B(z)$ is an all-zero filter and the input-output relationship is

$\mathrm{x}(\mathrm{n})=\sum_{\mathrm{k}=0}^{\mathrm{q}} \mathrm{b}_{\mathrm{k}} \mathrm{w}(\mathrm{n}-\mathrm{k})$

\section{ARMA Model}

In this model, the linear filter $\mathrm{H}(\mathrm{z})=\frac{\mathrm{B}(\mathrm{z})}{\mathrm{A}(\mathrm{z})}$ has finite zeros and poles, is a pole-zero filter in the z-plane and the difference equation is

$\mathrm{x}(\mathrm{n})+\sum_{\mathrm{k}=1}^{\mathrm{p}} \mathrm{a}_{\mathrm{k}} \mathrm{x}(\mathrm{n}-\mathrm{k})=\sum_{\mathrm{k}=0}^{\mathrm{q}} \mathrm{b}_{\mathrm{k}} \mathrm{w}(\mathrm{n}-\mathrm{k})$

The inverse system for generating the innovation process $\mathrm{x}(\mathrm{n})$ is also a pole-zero system of the form $\frac{1}{\mathrm{H}(\mathrm{z})}=\frac{\mathrm{A}(\mathrm{z})}{\mathrm{B}(\mathrm{z})}$.

\section{Prewhitening using Dechirping Transformation}

The conventional prewhitener methods depend on the local stationary of the LFM reverberation. The assumption of stationary within each block is doubtful, due to reverberation is produced by the multiple reflections and diffusions of the transmitted chirp signal on a large number of scatters and boundaries in the water. Due to these difficulties, affecting the accuracy of the spectral estimation and degrading the whitening performance. In this paper, the first method proposes a dechirping transformation based prewhitening using parametric spectral estimation AR, MA and ARMA models. This transformation is used to convert the LFM signal frequency into tonal-like frequency in each block [2]. A linear frequency modulated signal is in equation form [3]

$x(t)=e^{j 2 \pi\left(\left(f_{c}-\frac{B_{W}}{2}\right) t+\frac{B_{W}}{(2 T)} t^{2}\right)} \equiv e^{j 2 \pi\left(f_{0} t+\frac{m t^{2}}{2}\right)}$

for $0 \leq \mathrm{t} \leq 1$

where bandwidth is $B_{w}$, duration is $T$, central frequency is $f_{c}$, starting frequency is $f_{0}$, frequency modulating coefficient (or) frequency chip rate $m=\frac{B_{w}}{T}$, the dechirped signal

$\mathrm{x}_{\mathrm{D}}(\mathrm{t})=\mathrm{x}(\mathrm{t}) * \mathrm{e}^{\left(-\mathrm{j} 2 \pi \frac{\mathrm{mt} \mathrm{t}^{2}}{2}\right)}$

The concept of the dominant LFM reflection signals due to scatterers and boundaries in the water, where the length of the pulse is $T_{p}$ and the bandwidth is $B_{w}$ using dechirping transformation is interpreted in Figure 1[2].

The proposed prewhitening with dechirping transformation is summarized as the following procedure:

- Divide the received LFM reverberation data into blocks using a sliding window.

- Apply the dechirping transformation for current and adjacent block data. 
- To whiten the current block data extract the AR coefficients $\left\{1, \mathrm{a}_{1}, \ldots, \mathrm{a}_{\mathrm{p}}\right\}_{\mathrm{D}}, \mathrm{MA}$ coefficients $\left\{1, \mathrm{~b}_{1}, \ldots \ldots, \mathrm{b}_{\mathrm{q}}\right\}_{\mathrm{D}}$ and ARMA coefficients $\left(\mathrm{p}^{\text {th }}, \mathrm{q}^{\text {th }}\right)$ from modeling the reference of previous or next block data.

- From these AR, MA and ARMA coefficients, define the inverse filter for all proposed models

- Apply the inverse filter to $x_{D}(t)$, then obtain the whitened signal $w_{D}(t)$

- Because $w_{D}(t)$ is dechirped, rechirp it to restore its original state and whitened output $w(t)$ is obtained.

- Apply matched filter to detect a target.
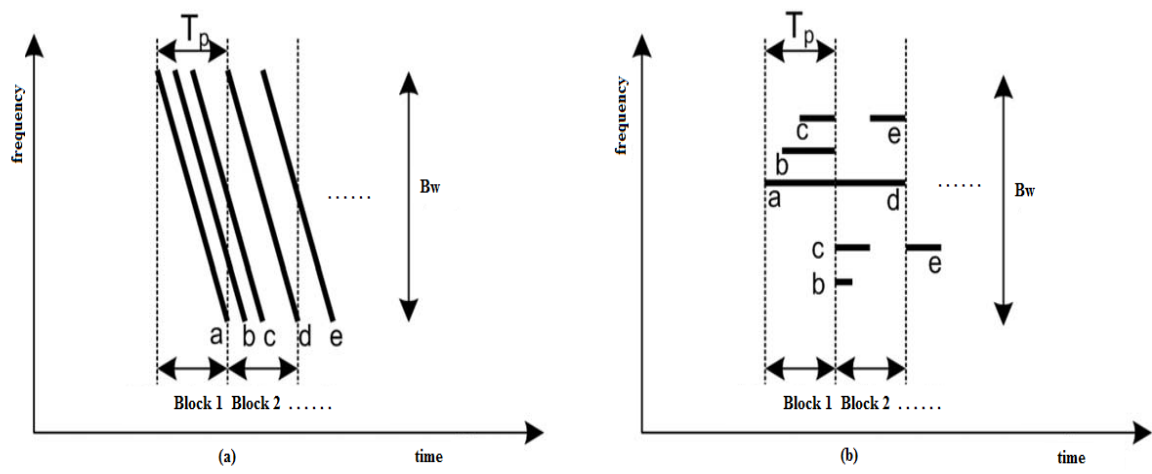

\section{Figure 1. (a) Before Dechirping Time and Frequency Characteristic of LFM Reverberation (b) After Dechirping Time and Frequency Characteristic of LFM Reverberation}

\section{Prewhitening using Fractional Fourier Transform}

In this paper, the second method proposes FrFT based prewhitening using parametric spectral estimation AR, MA and ARMA models. Principle concept of LFM reverberation with FrFT is interpreted in Figure 2. The LFM reverberation is expressed by overlap-add of multiple LFM reflection signals. The LFM signal equation is expressed in the earlier section. As a generalization of the ordinary Fourier Transform (FT), the FrFT is stronger in theory and more flexible in signal processing applications. FrFT is one of the linear transformations generalizing the Fourier transform. The FrFT is a linear operator and hence will not be influenced by the cross terms even if multiple and moving targets exist. To process the chirp signals, FrFT is better than the conventional Fourier Transform [4, 5]. The transform absorbs the chirp parameters in its kernel by a parameter $\alpha$, when the magnitude response of FrFT reaches its maximum, and the axis of rotation is matched to the chirp rate of the signal. This process is known as transform optimization. The Fourier transform is expressed as [3]

$Y_{\alpha}(f)=\int_{-\infty}^{\infty} B_{\alpha}(f, t) y(t) d t$,

$y(t)=\int_{-\infty}^{\infty} Y_{\alpha}(f) B_{-\alpha}(f, t) d f$

where $\mathrm{B}_{\alpha}(\mathrm{f}, \mathrm{t})$ is the transform kernel, $\mathrm{t}$ is the time and $\mathrm{f}$ is the frequency.

$B_{\alpha}(f, t)=\exp (-i 2 \pi f t)$

The FrFT is defined by modifying the Fourier transform kernel to the form 
$\mathrm{B}_{\propto(\mathrm{a}, \mathrm{b})}=\mathrm{R}_{\propto} \exp \left[\mathrm{i} \pi\left(\mathrm{a}^{2} \cot (\propto)-2 \mathrm{ab} \csc (\propto)+\mathrm{b}^{2} \cot (\propto)\right)\right]$

where $\mathrm{R}_{\propto}=\exp \left[-\frac{\mathrm{i} \pi \operatorname{sgn}(\sin (\alpha))}{4}+\frac{\mathrm{i} \propto}{2}\right] *|\sin (\propto)|^{-\frac{1}{2}}$

$\mathrm{a}$ and $\mathrm{b}$ defines the axes of the fractional domain and $\propto=\frac{\mathrm{a}_{\mathrm{opt}} * \pi}{2}$ is the rotation angle.

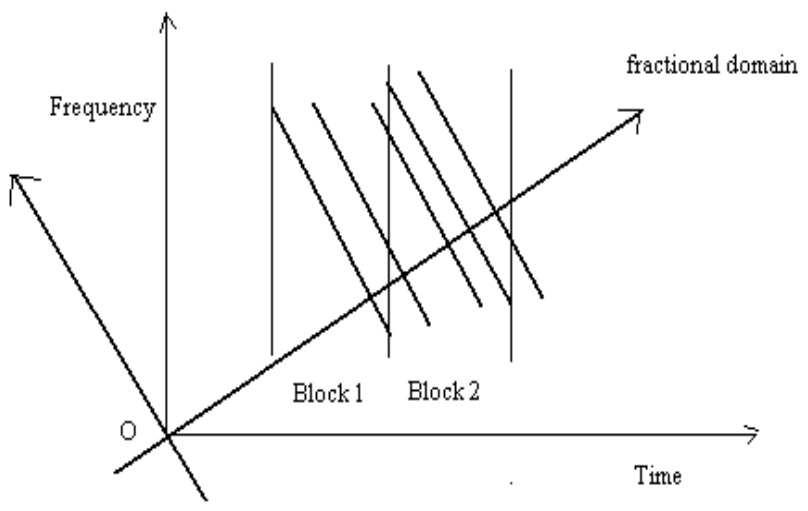

Figure 2. Principle Concept of LFM Reverberation with FrFT

The proposed prewhitening with Fractional Fourier Transform is summarized as the following procedure:

- Calculate the optimal order value $\mathrm{a}_{\text {opt }}$ with respect to the transmitted signal using equation 10

$\mathrm{a}_{\mathrm{opt}(\text { discrete })}=-\frac{2}{\pi} \tan ^{-1}\left(\frac{\Delta \mathrm{f} / \Delta \mathrm{t}}{\mathrm{m}}\right)=-\frac{2}{\pi} \tan ^{-1}\left(\frac{\frac{\mathrm{f}_{\mathrm{s}}^{2}}{\mathrm{~N}}}{\frac{\mathrm{B}_{\mathrm{w}}}{\mathrm{T}}}\right)=-\frac{2}{\pi} \tan ^{-1}\left(\frac{\mathrm{f}_{\mathrm{S}}^{2} * \mathrm{~T}}{\mathrm{~B}_{\mathrm{w}} * \mathrm{~N}}\right)$

where $\Delta \mathrm{f}=\mathrm{f}_{\mathrm{s}} / \mathrm{N}$ and $\Delta \mathrm{t}=1 / \mathrm{f}_{\mathrm{s}}$, bandwidth is $\mathrm{B}_{\mathrm{w}}$, chip rate $\mathrm{m}=\frac{\mathrm{B}_{\mathrm{w}}}{\mathrm{T}}$ and number of time samples in the waveform is $\mathrm{N}$.

- Divide the LFM reverberation data into blocks using a sliding window.

- Apply FrFT transform for each block of the current data

- Whitening the current block using parametric AR, MA and ARMA models in fractional domain.

- Recover the whitened signal by combining all blocks data in fractional domain

- Apply matched filter to detect a target.

\section{Results}

To suppress the reverberation using proposed dechirping transformation and FrFT based prewhitening methods, sample LFM reverberation data was assumed in an underwater environment. The transmitted signal was LFM with bandwidth is $2.5 \mathrm{kHz}$, center frequency is $3.25 \mathrm{kHz}$, and sampling frequency is $18 \mathrm{kHz}$ respectively. LFM reverberation data were created by combining the following three conditions and white Gaussian noise level at $-20 \mathrm{db}$ on the transmitted signal. In the first condition assumed target is moving with a speed is 10 nautical mile, amplitude attenuation is 1 and pulse duration is $0.13 \mathrm{sec}$. In the remaining conditions assumed target is moving with a speed is 30, 50 nautical miles, amplitude attenuation is $1.5,2$ and pulse duration is $0.1 \mathrm{sec}$ respectively. In this experiment, the AR model order was selected as $(p=20)$ and MA 
model order was selected as $(\mathrm{q}=4)$ and ARMA model order was selected as $(20,4)$. Time domain plot of LFM reverberation data is shown in Figure 3. Graph was plotted between time and amplitude of the signal.

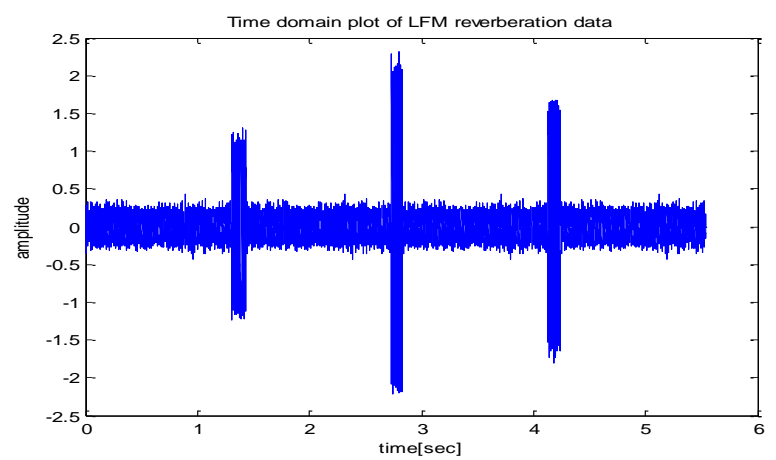

Figure 3. Time Domain Plot of LFM Reverberation Signal

The 3-D mesh plot of prewhitening using with and without conventional methods is shown in Figure 4. 3-D mesh was plotted between time, frequency and energy of the signal.

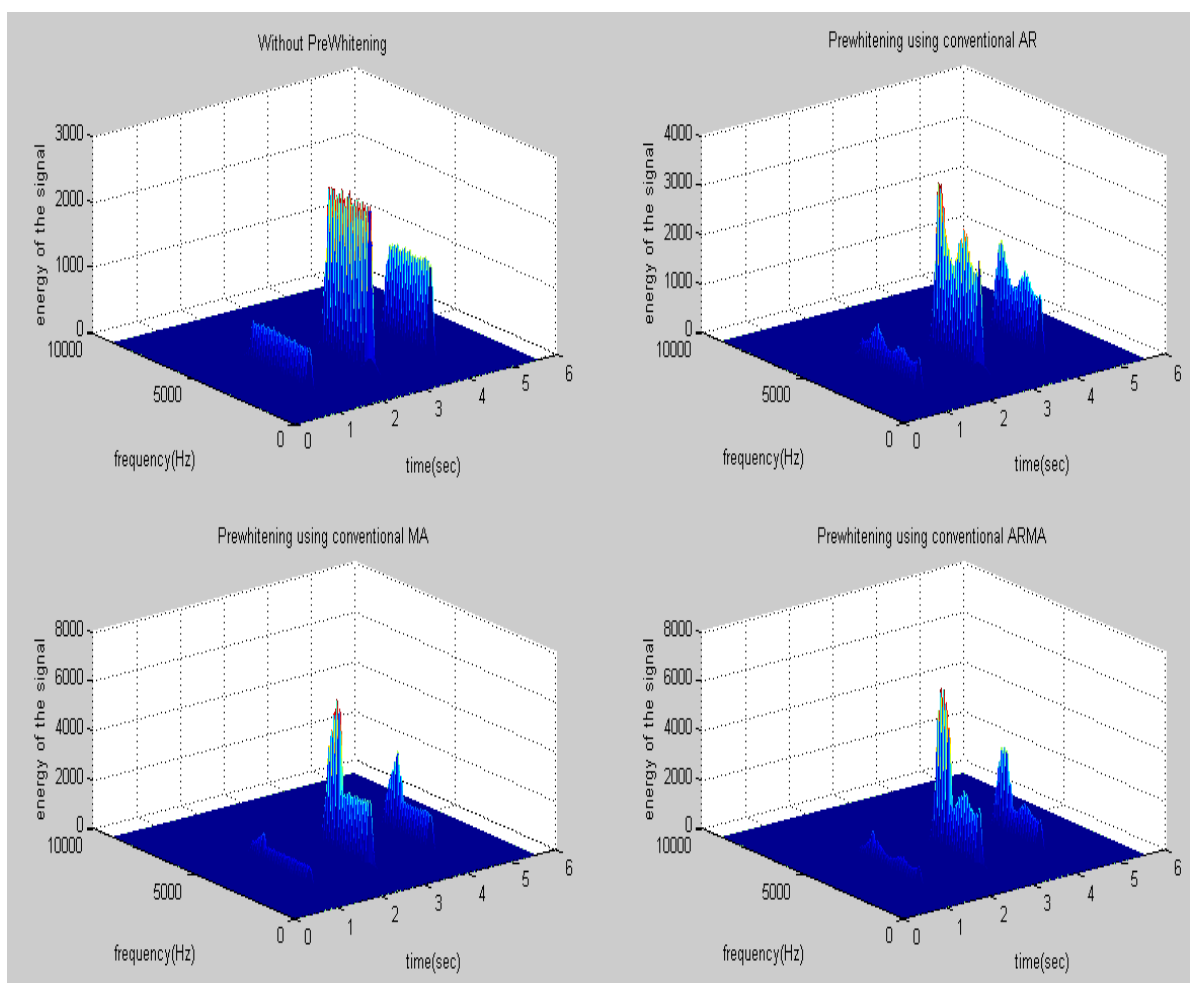

Figure 4. Prewhitening Results using Conventional Methods (a) Without Prewhitening (b) Prewhitening using AR model (c) Prewhitening using MA Model (d) Prewhitening using ARMA Model

The Matched Filter output after the conventional based AR, MA and ARMA prewhitening models are shown in Figure 5. This shows that the conventional based MA, ARMA prewhitening models work well for a stationary target and/or a moving target as compared with conventional based AR prewhitening model. 

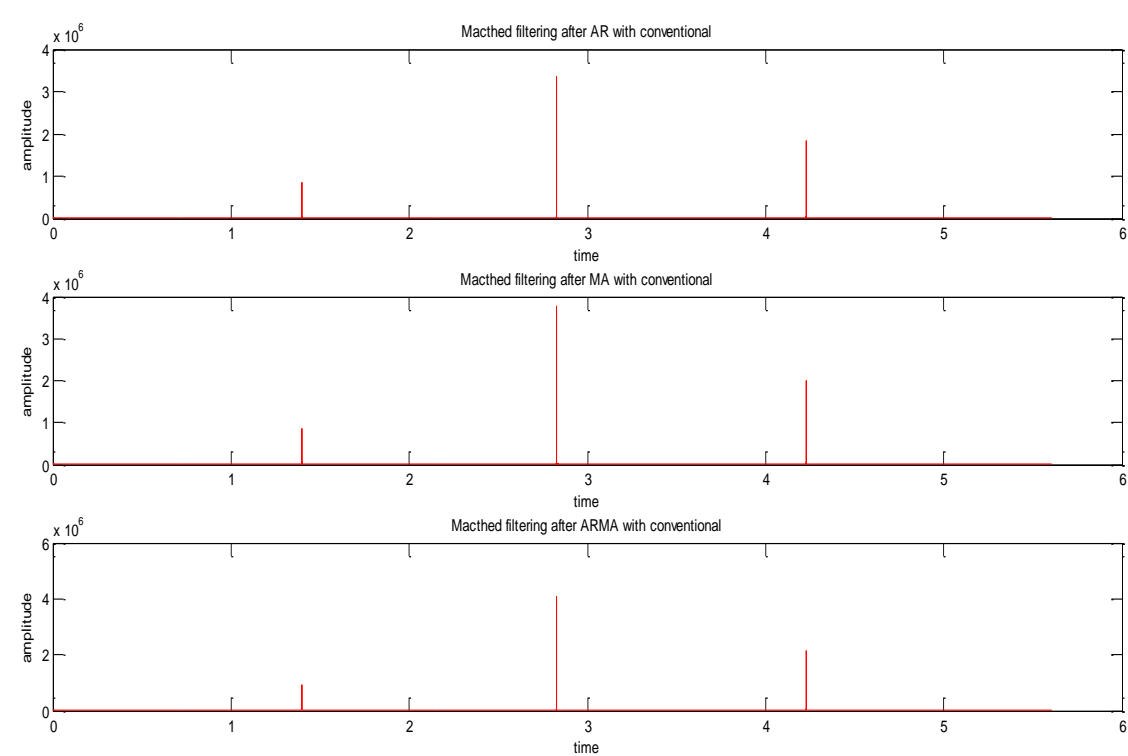

Figure 5. Matched Filter Results after Applying Conventional based Prewhitening AR, MA and ARMA Methods

The 3-D mesh plot of prewhitening using with and without dechirping transformation is shown in Figure 6. The 3-D mesh was plotted between time, frequency and energy of the signal.

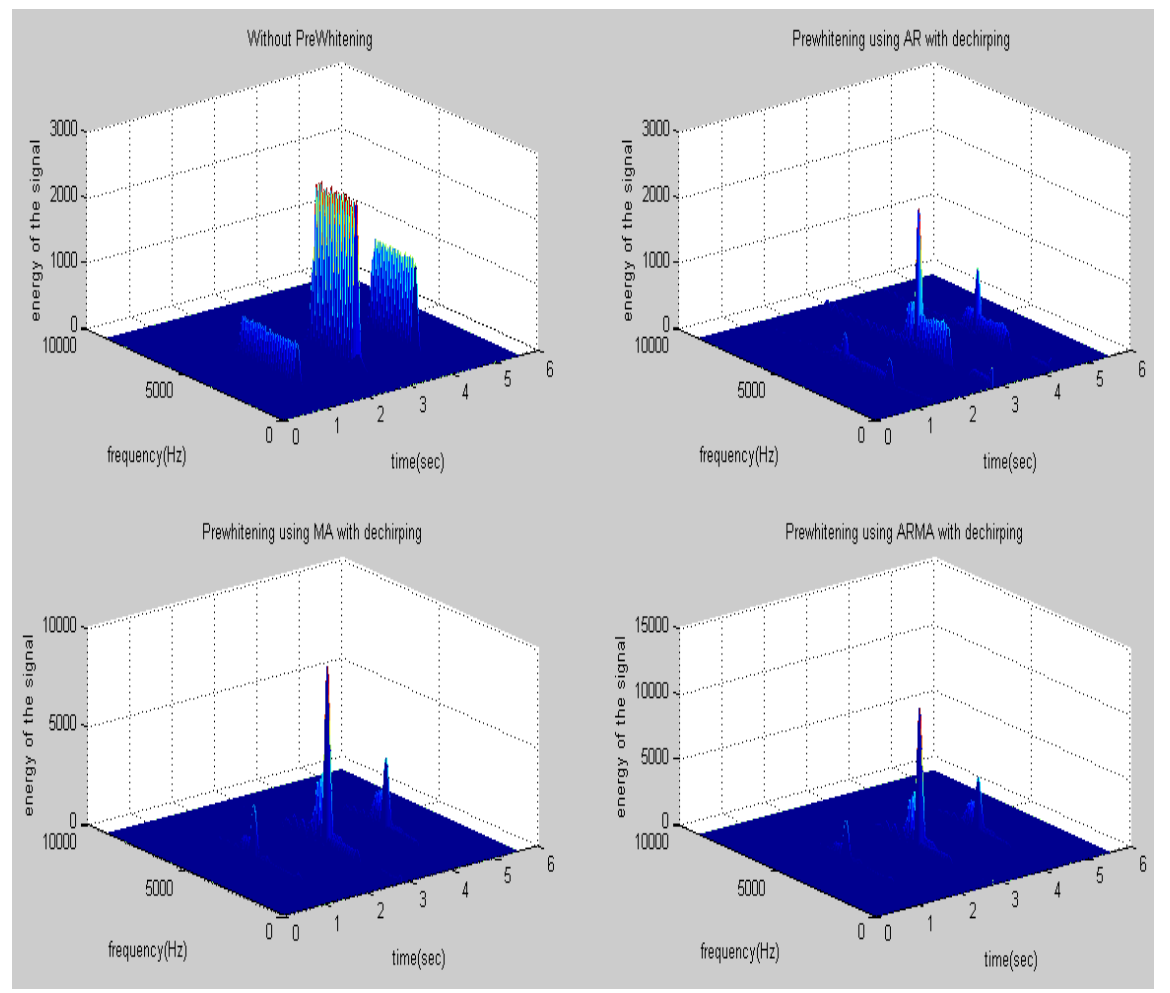

Figure 6. Proposed Dechirping Transformation based Prewhitening Results (a) Without Prewhitening (b) Prewhitening using AR Model (c) Prewhitening using MA Model (d) Prewhitening using ARMA Model 
The Matched Filter output after the dechirping based AR, MA and ARMA prewhitening models are shown in Figure 7. The result reveals each target peak prominently due to LFM reverberation from all directions. This shows that the proposed dechirping based MA, ARMA prewhitening models work well for a stationary target and/or a moving target as compared with dechirping based AR prewhitening model.
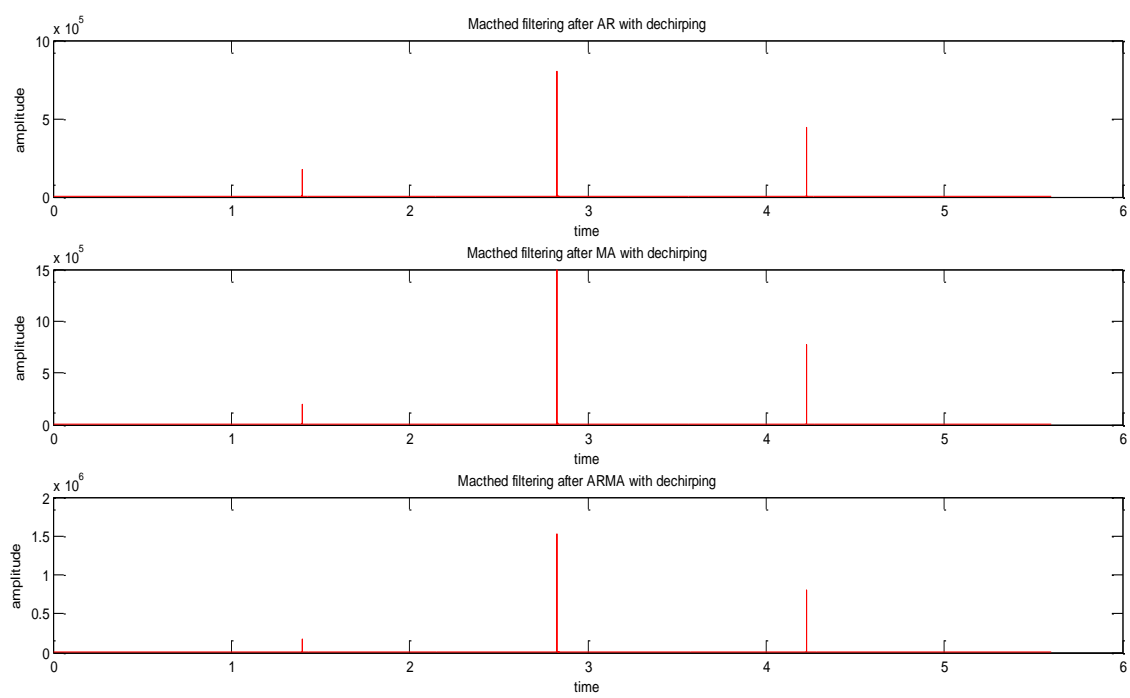

\section{Figure 7. Matched Filter Results after Applying Proposed Dechirping Transformation based Prewhitening AR, MA and ARMA Methods}

The 3-D mesh plot of prewhitening using with and without FrFT transform is shown in Figure 8. The 3-D mesh was plotted between time, frequency and energy of the signal.

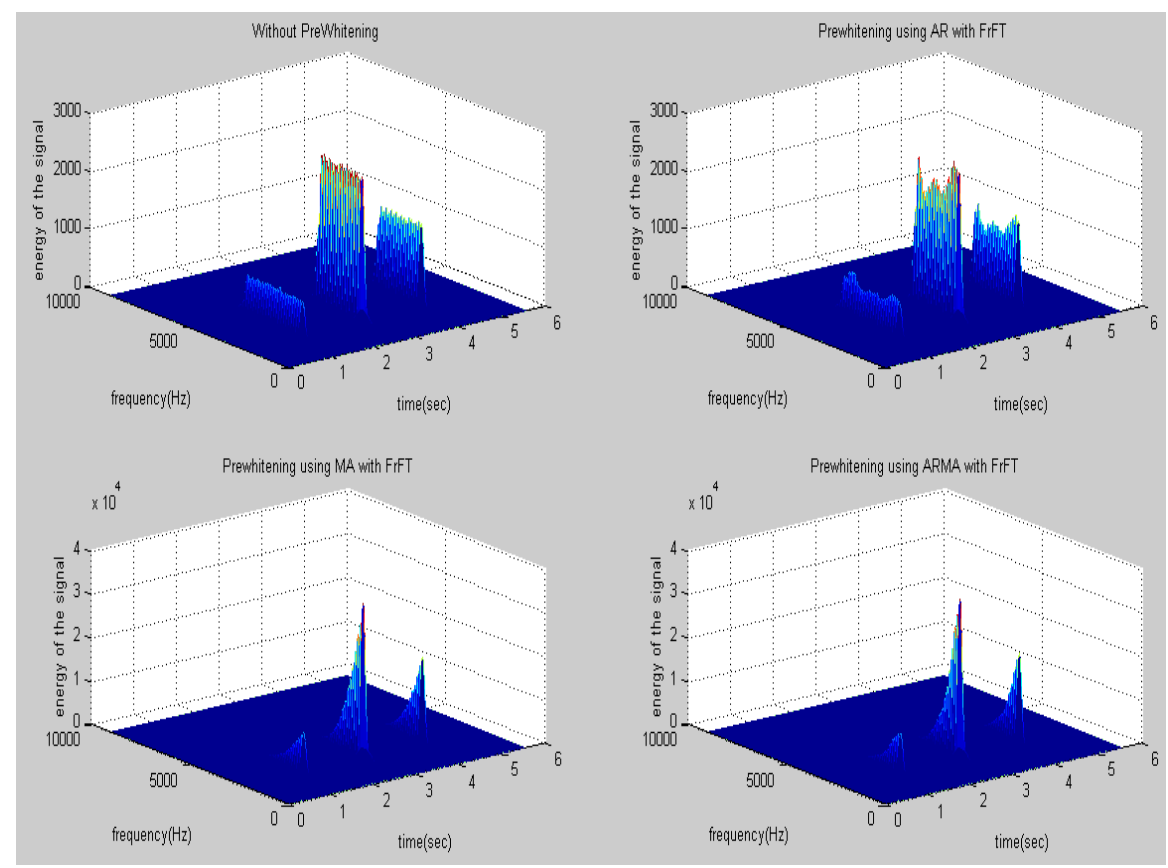

Figure 8. Proposed FrFT Transform based Prewhitening Results (a) Without Prewhitening (b) Prewhitening using AR model (c) Prewhitening using MA Model (d) Prewhitening using ARMA Model 
The Matched Filter output after the FrFT based AR, MA and ARMA prewhitening models are shown in Figure 9. The result reveals each target peak prominently due to LFM reverberation from all directions. This shows that the proposed FrFT based MA, ARMA prewhitening models work well for a stationary target and/or a moving target as compared with FrFT based AR prewhitening model.
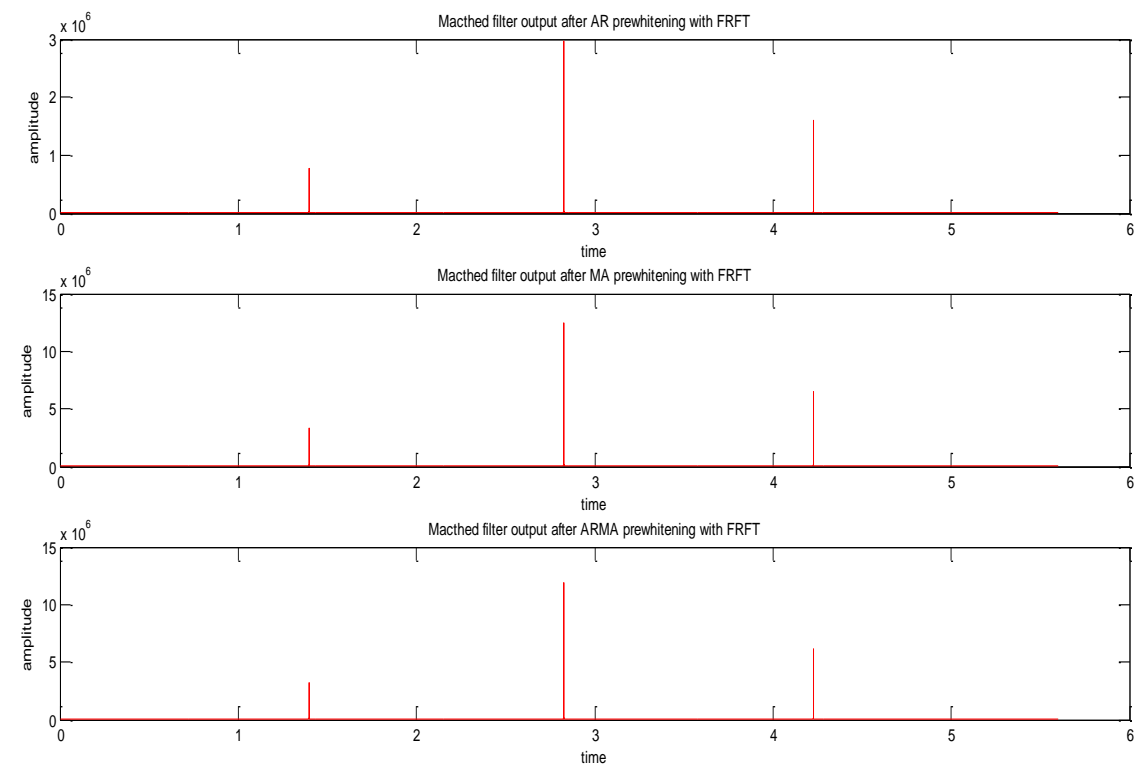

\section{Figure 9. Matched Filter Outputs after Applying Proposed FrFT Transform based Prewhitening AR, MA and ARMA Methods}

From the simulation results of LFM reverberation suppression it is clearly observed that the proposed dechirping and FrFT based prewhitening models will gives better performance as compared with the conventional based prewhitening models. And also it is clearly observed that proposed MA, ARMA based prewhitening models will gives better performance as compared with AR based prewhitening models.

\section{Conclusion}

In this paper, the study of performance analysis of LFM reverberation suppression using conventional based, dechirping transformation based and FrFT based AR, MA and ARMA prewhitening methods has been explored. From simulation results with LFM reverberation data proves that the proposed dechirping transformation based and FrFT based prewhitening models will give better reverberation suppression as compared with conventional based prewhitening models. It is clearly observed that the proposed dechirping transformation based and FrFT based MA and ARMA prewhitening models will provide an improved whitening performance as compared with AR prewhitening model.

\section{References}

[1] R. Wang, J. Huang, T. Ma and Q. Zhang, "Improved space time prewhitener for linear frequency modulation reverberation using fractional Fourier transform”, Acoustical Society of America, Journal of Acoust. Soc. Am., (2010), vol. 128, no. 6, pp. 361-365.

[2] B. W. Choi, E. H. Bae, J. S. Kim and K. K. Lee, "Improved prewhitening method for linear frequency modulation reverberation using dechirping transformation", Journal of Acoust. Soc. Am., vol. 123, no. 3, (2008), pp. 21-25. 
[3] D. M. J. Cowell and S. Freear, "Separation of Overlapping Linear Frequency Modulated (LFM) Signals Using the Fractional Fourier Transform", IEEE Transactions on Ultrasonics, Ferroelectrics, and Frequency Control, vol. 57, no. 10, (2010), pp. 2324-2333.

[4] E. Sejdic, I. Djurovic and L. Jubisa Stankovic, "Fractional Fourier transform as a signal processing tool: An overview of recent developments", Elsevier, Signal Processing, vol. 91, no. 6, (2011), pp. 13511369.

[5] V. Ashok Narayanan and K. M. M. Prabhu, "The fractional Fourier transform: theory, implementation and error analysis", Elsevier Microprocessors and Microsystems, vol. 27, (2003), pp. 511-521.

[6] V. Camillet, P. Amvlard and G. Jourdain, "Detection of phase or frequency modulated signals in reverberation noise", Journal of Acoust. Soc. Am., vol. 105, no. 6, (1999), pp. 3375-3389.

[7] S. Kay and J. Salisbury, "Improved active sonar detection using autoregressive prewhiteners," J. Acoust. Soc. Am., vol. 87, no. 4, (1990), pp. 1603-1611.

[8] W. J. Chen, H. Sun and J. J. Zhu, "A method for detecting of the target echo in reverberation noise", Proceedings of IEEE International Ultrasonics Symposium, BICC, (2008), pp. 1445-1448.

[9] P. Stoica and R. Moses, "Spectral Analysis of Signals", Prentice Hall, Upper Saddle River, New Jersey, (2005).

[10] J. G. Proakis and D. G. Manolakis, "Digital Signal Processing", Pearson Education, Fourth edition, (2007).

\section{Authors}

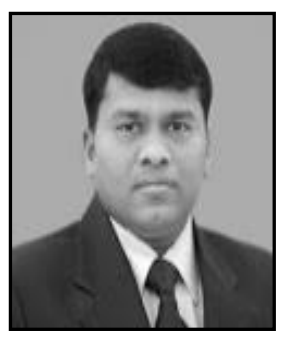

Jami Venkata Suman has completed Bachelor's degree from Tontadarya College of Engineering, under VTU, Belgaum and Karnataka state. He Received Master's degree in VLSI System Design from Annamacharya Institute of Technology and Sciences, Rajampet, under JNTU, Hyderabad and Master of Business Administration in HRM and MRKT from Andhra University, Visakhapatnam, Andhra Pradesh. He is pursuing PhD (Part-Time) in Signal Processing area at GITAM Institute of Technology, Visakhapatnam. He is currently working as an Assistant Professor in the Department of Electronics and Communication Engineering at GMR Institute of Technology, Rajam. Major Research areas include Signal Processing Techniques in Radar \& Sonar and Low Power VLSI Design. He is Life Member of ISTE.

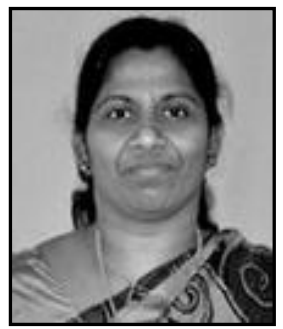

J. Beatrice Seventline is graduated in Electronics and Communication Engineering from Bharathiar University during 1991. She obtained her Master's Degree in Microwave and Optical Engineering from Madurai Kamaraj University during 1994. She received the Ph.D degree in the field of Radar Signal Processing from Andhra University in the year 2012. Presently, she is Associate Professor and Assistant Principal, Department of ECE, GITAM Institute of Technology, GITAM University, Visakhapatnam, India. Her research interests are Signal processing techniques in Radar, Sonar, Spread spectrum communication and Microwaves. 